livraisons

d'Histoire

de l'Architecture

\section{Livraisons de l'histoire de l'architecture}

26 | 2013

Les ministres et les arts

\title{
Philibert Orry et l'embellissement du territoire autour de l'Instruction de 1738 : genèse d'un paysage routier et urbain
}

Philibert Ory and the territory's embellishment according to the Instruction of 1738 : genesis of a road and urban landscape

Philibert Orry und die Verschönerung des Landes in dem Zusammenhang der Ermittlung im Jahr 1738: die Schaffung einer städtischen Landschaft

\section{Dominique Massounie}

\section{OpenEdition}

Journals

Édition électronique

URL : http://journals.openedition.org/lha/338

DOI : $10.4000 /$ /ha.338

ISSN : 1960-5994

Éditeur

Association Livraisons d'histoire de l'architecture - LHA

Édition imprimée

Date de publication : 10 décembre 2013

Pagination : 89-104

ISSN : 1627-4970

Référence électronique

Dominique Massounie, "Philibert Orry et l'embellissement du territoire autour de l'Instruction de 1738 genèse d'un paysage routier et urbain », Livraisons de l'histoire de l'architecture [En ligne], 26 | 2013, mis en ligne le 10 décembre 2015, consulté le 19 avril 2019. URL : http://journals.openedition.org//ha/338 ; DOl : 10.4000//ha.338 


\section{PHILIBERT ORRY ET L'EMBELLISSEMENT DU TERRITOIRE AUTOUR DE L'INSTRUCTION DE 1738 : GENESE D'UN PAYSAGE ROUTIER ET URBAIN}

Les routes et les avenues des grands chemins, bien unis, font la beauté des grandes et des petites villes, font l'ornement des provinces, facilitent le commerce dans le royaume ${ }^{1}$.

En mai 1737, un Mémoire sur la conduite du travail par corvée avait été imprimé pour être envoyé à tous les intendants. Il fut suivi du Mémoire instructif sur la réparation des chemins signé le 13 juin 1738 par Philibert Orry, contrôleur général des finances: ce texte généralisait la corvée pour les travaux routiers. L'Instruction de 1738 est considérée comme le texte fondateur de la création des grands chemins qui devinrent routes impériales puis nationales et qui sont, dans la mémoire collective, ces interminables lignes droites tracées au milieu des champs ou au cœur des forêts, traversant quelques villes et des bourgades - rues, routes bordées de grands arbres, dont la plupart devait disparaître à partir des années 70 en raison de la gravité des accidents que provoquait la moindre sortie de route des nouveaux bolides.

Les historiens et géographes ont depuis longtemps prêté attention à ce phénomène exceptionnel de la seconde moitié du XVIII ${ }^{\mathrm{e}}$ siècle qu'est la modernisation des routes du royaume de France, spectaculaire au point que notre meilleur ennemi, Arthur Young, y voyait là, en 1787, une révolution : "Si les Français n'ont pas d'agriculture à nous montrer, ils ont des routes; rien de plus magnifique, de mieux tenu que celle qui traverse un beau bois, propriété de celle de M. Neuvillier ; on croirait voir une allée de parc. Et certes, tout le chemin à partir de la mer est merveilleux : c'est une large chaussée aplanissant les montagnes au niveau des vallées $[\ldots]^{2}$. Les magnifiques chemins que nous avons suivis, si fort au-dessus de tout ce que nous avons vu en France $[. . .]^{3}$. Les routes sont d'admirables travaux. J'ai passé une tranchée dans le roc vif qui facilite une descente, elle a couté 90000 livres (3997 1. st.) pour quelques centaines de yards. Les trois lieues et demie de Sigean à Narbonne coute 1800000 livres (78 750 l. st.). On a fait des folies, des

1. Henri (dit parfois Hubert) Gautier, Traité de la construction des chemins, où il est parlé de ceux des Romains, et de ceux des Modernes, de leur figure, de leur matière et de leur disposition dans toute sorte de lieux, Toulouse, J. Dominique Camusat, 1693, voir l'édition de Paris, Laporte, 1778, préface.

2. Arthur Young, Voyages en France pendant les années 1787, 1788, 1789, [Paris, 1794, première édition en anglais 1792] ; Paris, Guillaumin et $C^{\text {ie }}$, 1882, t. I, p. 9-10.

3. Ibid., p. 29. 
sommes énormes ont été employées au nivellement des pentes les plus douces. Les chaussées sont en remblai, avec un mur de soutènement de chaque côté, formant une masse artificielle solide, traversant les vallées à la hauteur de six, sept et huit pieds, et n'ayant pas moins de cinquante pieds de large. Il y a un pont d'une seule arche dont la chaussée est vraiment quelque chose d'admirable [...] un tiers de la chaussée est battue [...]. » Comme d'ordinaire, l'Anglais qualifie d'injuste la contribution qui remplace la corvée en Languedoc, pays d'État, et de terminer par l'une de ces images contrastées qui firent le succès de ce récit : "Le 24 [juillet 1787]. Des femmes sans bas, beaucoup même sans soulier ; mais si leurs pieds sont pauvrement couverts, il leur reste la superbe consolation de les poser sur une chaussée grandiose ; la nouvelle voie a cinquante pieds de large, plus cinquante autres déblayés pour lui faire place ${ }^{4}$."

Eugène Vignon au XIX $\mathrm{X}^{\mathrm{e}}$ siècle a ouvert la voie à l'étude des routes en France ${ }^{5}$; Bernard Lepetit et Guy Arbellot, pour ne citer qu'eux, ont contribué de manière importante à la connaissance du réseau viaire et de la cartographie du royaume ${ }^{6}$. Dernièrement, Stéphane Blond a apporté sa pierre à la compréhension de la production des fameux atlas de Trudaine $e^{7}$. Quelques historiens de l'art ont déjà souligné le rapport entre la transformation des grands chemins et celle des villes: ils ont emprunté au vocabulaire des ingénieurs le nom de "traverse ", qui désigne la route principale qui va d'une porte à une autre et qui fait l'objet de redressements et d'élargissement, soit d'alignement, pour qualifier une rue susceptible d'embellissement par l'ordonnancement des façades, la création d'une place ou encore la présence d'une ou de plusieurs édifices publics ${ }^{8}$. Pierre Bodineau puis Pierre Pinon ont étudié le déroulement des quelques opérations d'alignement, tandis que Yoann Brault, auquel on doit les travaux les plus développés sur la question de la transformation de l'abord des villes aux XVII ${ }^{e}$ et XVIII ${ }^{e}$ siècles, a notamment exposé les diverses raisons de la reconstruction des portes de ville, parmi lesquelles le "formidable effort routier entrepris par le corps des ingénieurs des Ponts et

4. Ibid., p. 55-56.

5. Eugène Vignon, Études historiques sur l'administration des voies publiques en France aux XVII et XVIII ${ }^{e}$ siècles, Paris, Dunod, 1862, 3 vol.; l'auteur a édité de nombreuses sources à l'appui de son travail.

6. Bernard Lepetit, Guy Arbellot, Atlas de la Révolution française. Routes et communications, Paris, éditions de l'ÉHESS, 1987, 91 p. ; Bernard Lepetit, Les Villes dans la France moderne, 1740-1840, Paris, Albin Michel, 1988, 490 p. ; Guy Arbellot, Autour des routes de postes. Les premières cartes routières de la France, XVII - -XIX $X^{e}$ siècles, Paris, BnF, Musée de la Poste, 1992, 181 p. et 52 cartes ; "La grande mutation des routes de France au XVIII siècle", Annales. Économie, sociétés, civilisations, $28^{\mathrm{e}}$ année, $\mathrm{n}^{\circ} 3,1973$, p. 765-791.

7. Stéphane Blond, L'Atlas de Trudaine: pouvoir, administrations et savoirs techniques (vers 1730-vers 1780), thèse de doctorat sous la dir. de Daniel Nordman, ÉHESS, 2008 (à paraître aux éditions du CTHS).

8. Voir aussi Dominique Massounie, "Autour de 1759: deux décennies de renouvellement urbain ", Le Public et la politique des arts au Siècle des Lumières, Christophe Henry, Daniel Rabreau dir., actes du colloque de 2009, Paris-Bordeaux, Centre Ledoux et William Blake, 2011, p. 181-190 (collection Annales du Centre Ledoux, t. VII). 
chaussées ${ }^{9}$ ". Antoine Picon, enfin, a démontré l'intérêt des ingénieurs des Ponts et chaussées pour l'architecture et parfaitement expliqué leur mission : équiper et gérer un territoire que l'on s'efforce de cartographier depuis 1670: "Se démarquant de l'architecte, homme d'un projet limité dans l'espace et dans le temps, l'ingénieur pourra alors élaborer des politiques d'aménagement globales ${ }^{10}$."

Prêter au contrôleur général des finances l'intention d'embellir n'est sans doute qu'une interprétation abusive du texte de l'Instruction, même si le ministre exerce conjointement, après la mort du duc d'Antin en 1736, la fonction de directeur général des Bâtiments du roi. Avant l'abbé Terray, à la Motte-Tilly, près de Nogent-sur-Seine ${ }^{11}$, Orry embellit la demeure familiale de La Chapelle-Godefroy, entre Nogent et Pont-sur-Seine, sur la grande route de Paris en Champagne. Les jardins tout comme la demeure et son décor témoignent de la magnificence des lieux, aujourd'hui détruits. Deux planches des Atlas de Trudaine figurent le domaine : le château, acquis par son père, a été entièrement reconstruit au tout début du XVIII ${ }^{\mathrm{e}}$ siècle, au centre d'un remarquable jardin régulier ${ }^{12}$. Dans les années 1730, Philibert Orry contribue au renouveau de la peinture d'histoire en s'adressant à Charles-Joseph Natoire pour exécuter dans sa demeure champenoise trois cycles respectivement consacrés à l'histoire des dieux, de Clovis, de Télémaque, et un ensemble figurant les Saisons ${ }^{13}$. Deux artistes réputés ont fait son portrait : Hyacinthe Rigaud, vers 1737, le représente dans l'exercice de ses fonctions, tout comme Maurice-Quentin de La Tour ${ }^{14}$. Mais affirmer que Philibert Orry avait une quelconque intention d'embellir le territoire en favorisant la modernisation des routes par la généralisation de la corvée serait un abus. En revanche, au-delà de la question de l'intention, on est en droit d'examiner les répercussions éventuelles d'un règlement relatif aux voies publiques sur les territoires traversés, villes et campagnes. La nature de la chaussée, sa largeur, l'aménagement de trottoirs, l'installation de garde-corps, d'un éclairage public et l'érection d'un monument sur les ponts, le franchissement d'une rivière ou d'un ravin, la plantation d'arbres, l'alignement, l'infléchissement d'une direction, la création de carrefours sont autant d'interventions susceptibles de modifier l'environnement paysager et urbain.

9. Pierre Bodineau, L'Urbanisme dans la Bourgogne des Lumières, Dijon, publications du centre Georges Chevrier pour l'histoire du droit, 1986, 284 p.; Pierre Pinon, "Alignements, percées et lotissements en France au XVIII ${ }^{e}$ siècle ", De l'esprit des villes. Nancy et l'Europe urbaine au siècle des Lumières, 1720-1770, Alexandre Gady, Jean-Marie Pérouse de Montclos dir., Versailles, éditions Artlys, p. 162-172; Yoann Brault, "Ville déclose, ville ouverte", ibid., p. 142 à 151; Naissance d'une promenade urbaine : le boulevard (1670-1676). Identité de la ville absolutiste, problématiques, mémoire de maîtrise sous la dir. de Daniel Rabreau, université Paris 1-PanthéonSorbonne, 1998; thèse à venir élargissant le sujet jusqu'à la fin de l'Ancien Régime.

10. Antoine Picon, Architectes et ingénieurs au siècle des Lumières, Marseille, Parenthèses, 1988, p. 97.

11. Voir dans le même volume l'article de Rose-Marie Chapalain.

12. Arch. nat., $\mathrm{F}^{14} 8445$, généralité de Champagne, route $\mathrm{n}^{\circ} 22,18$ cartes, cartes 15 et 16 .

13. Susanna Caviglia-Brunel, Charles-Joseph Natoire, Paris, Arthena, 2012, 581 p.

14. Huile sur toile et pastel respectivement conservés au château de Versailles et au musée du Louvre. 
On a coutume de faire figurer le Pont-Neuf au rang des plus belles créations architecturales et urbaines de la période moderne : une large chaussée, presque une place publique entre les deux rives, bordée de trottoirs et de haltes munies de bancs, un pont - belvédère sur lequel la foule se presse lors des fêtes données sur le bassin royal, un chef-d'œuvre de stéréotomie. Au savoir des architectes succède au XVIII ${ }^{e}$ siècle la science des ingénieurs dont le travail autorise des nouvelles prouesses: il a été possible de construire là où les plus illustres architectes avaient échoués, comme à Moulins où les sables semblent n'avoir pas de fond, ou encore à Saumur où la Loire est si large, et la portée des arches audacieusement surbaissées ne cesse de croître. Ainsi, le pont demeure dans l'historiographie un programme d'ingénieur. Tout au plus forme-t-il avec la rue qui le prolonge à l'intérieur de la ville - ponts d'Orléans, de Tours, de Mantes - un même objet d'étude pour l'historien de l'art. Or, le pont comme la rue ne sont que le prolongement de la route, sur le fleuve et dans ou autour de la ville. Pourquoi alors ignorer que ce que détermine l'Instruction de 1738 et ne pas chercher à examiner le programme plus vaste et unique de la route entrant dans la ville au regard de ces prescriptions? De la même manière, l'importance des règlements dans la transformation de l'habitat a déjà été démontrée, et, si des principes bien différents de ceux de l'architecture classique sont le plus souvent à l'origine des interdictions et des recommandations - sécurité et salubrité -, leur respect, comme leur détournement, a des conséquences notoires sur l'architecture: choix des matériaux et élévation des immeubles ${ }^{15}$. Qu'en est-il de l'architecture des ponts et des portes au regard des conditions de leur établissement?

\section{Largeur et alignement des chaussées}

L'édit de juillet 1607 fixait la largeur des grands chemins royaux à 72 pieds $(23,33 \mathrm{~m})$, et prescrivait la conservation d'une largeur supérieure là où elle existait - cette cote correspond à l'emprise totale de la route. L'article troisième du même édit qui ordonnait "la coupe de tous les bois, épines et broussailles, qui se trouveront dans l'espace de 60 pieds $(19,44 \mathrm{~m})$ des grands chemins servant au passage des coches et carrosses publics, tant dans nos forêts que dans celles des ecclésiastiques, communautés, seigneurs et particuliers " ${ }^{16}$ aux frais du propriétaire, fixait la valeur finalement retenue pour trois siècles pour la partie carrossable uniquement. L'objet principal de ce texte étant de favoriser la circulation, quelle qu'elle soit, il prescrivait également le dégagement des voies bordant les rivières navigables à 24 pieds $(7,77 \mathrm{~m})$ au minimum, "pour chemin royal et traits de chevaux » et, avec défense de planter, clôturer ou faire sortie sur 30 pieds $(9,72 \mathrm{~m})$ de

15. Jean-François Cabestan, La Conquête du plain-pied. L'immeuble parisien au XVIII siècle, Paris, Picard, 306 p., 86 pl.

16. Henri Gautier, Traité de la construction des chemins, op. cit., p. 214 ; textes originaux reproduits en annexe par l'auteur. 
plus côté chemin de halage et $10(3,24 \mathrm{~m})$ de l'autre, ce qui équivaut à une emprise totale de 34 à 54 pieds $(11 \text { à } 17,50 \mathrm{~m})^{17}$. Il faut en effet distinguer la chaussée pavée ou empierrée, sur laquelle roulent généralement les voitures, d'éventuelles chaussées latérales, de terre, qui précèdent les fossés, puis des sortes de bermes, non carrossables, pratiquées encore au-delà, entre les fossés et les arbres, murs ou haies. Trois parties font ainsi l'objet de prescriptions, sous forme de lois ou d'instruction : la chaussée centrale, celle qui emprunte l'arche d'une porte triomphale, la ou bien les chaussées latérales, pour un usage déterminé, comme le halage, et enfin l'emprise totale de la route qui correspond aux limites de propriété.

En 1693, Henri Gauthier, ingénieur de la Province du Languedoc, proposait une manière précise de déterminer la largeur praticable d'une voie: le moyen de locomotion adopté, du carrosse au cheval, dictait la largeur. Il faut au simple piéton un pied et demi à 2 de passage, tandis qu'un cheval nécessite 3 à 4 pieds, un chariot, 7 à 8 pieds : ainsi varie la largeur des voies privées. Pour les chemins de traverse, deux chariots doivent s'y croiser de front : ils auront au moins 8 à 10 pieds, plutôt 14 à 15 pieds de large. Quant aux chemins royaux, le passage de quatre chariots de front les déterminent, entre 18 et 20 pieds, "mais comme il survient des rencontres imprévues, comme la file du bagage d'une armée, de troupes en marche, un débarquement ou un embarquement, ce qui peut supposer une grande quantité de voitures, l'arrangement et le dérangement d'une foire, ce qui suppose des foules de peuples et de voitures, et doivent faire connaître que suivant les occasions et les lieux, les grands chemins doivent avoir pour le moins 24 à 30 pieds de large, et ornés comme l'occasion le permettra et suivant la disposition des lieux ${ }^{18}$ ". Comme le souligne Arthur Young à la vieille de la Révolution, les grands chemins ont donc bien été surdimensionnés par rapport à l'usage ordinaire et ce, dès le $\mathrm{XVII}^{\mathrm{e}}$ siècle.

Une ordonnance des trésoriers généraux, en date du 17 décembre 1686, ordonnait ensuite que « tous les chemins allant de province en province, et de ville en ville " occuperaient 45 pieds et "les chemins allant de bourgs et villages aux villes " 30 pieds $^{19}$. En effet, entre le début du XVII ${ }^{e}$ siècle et l'arrêt du 3 mai 1720 , on observe des variations, souvent provinciales, mais, les chemins des Romains ayant jusqu'à 60 pieds de large ${ }^{20}$, c'est finalement cette mesure qui est généralisée au $\mathrm{XVIII}^{\mathrm{e}}$ siècle pour l'emprise totale. Cette largeur avait été adoptée dès août 1669, dans la fameuse Ordonnance des Eaux et forêts, pour les routes royales traversant les bois. Deux autres textes importants précèdent l'Instruction de 1738 : l'arrêt du 26 mai 1705 et celui du 3 mai 1720 . Le premier ordonne que "les ouvrages de pavé qui se feront de nouveau [...], et les anciens qui seront relevés seront conduits du plus droit alignement que faire se pourra ${ }^{21} »$. Le second se réfere au premier

17. Ibid., p. 215.

18. Ibid., p. 14-15.

19. Ibid., p. 225.

20. Ibid., p. 15, chapitre II, "Des grands chemins des modernes en général, de leurs chemins de traverse et de leurs chemins privés ».

21. Ibid., p. 239. 
pour renouveler la nécessité d'aligner l'ensemble des routes, pavées ou non. Le profil de la chaussée a été décrit dès 1705 : " il sera fait des fossés de 4 pieds de largeur sur 2 de profondeur [ $1,30 \mathrm{~m}$ sur $0,65 \mathrm{~m}$ ], à l'extrémité des chemins de terre qui sont de chaque côté du pavé [...], et lorsqu'il n'y aura point de chemins de terre déterminés, il en sera fait à 3 toises [ou 18 pieds, soit $5,83 \mathrm{~m}$ ] de distance du pavé de chaque côté dans lesdites grandes routes, et à 12 pieds $[3,89 \mathrm{~m}]$ dans les routes moins considérables, et ce, tant pour l'écoulement des eaux que pour conserver la largeur des chemins et les héritages riverains ${ }^{22}$. " $\mathrm{La}$ conservation des fossés est assurée par la défense de planter des arbres au moins à 3 pieds $(1 \mathrm{~m})$. L'arrêt du 3 mai 1720 clarifie encore les prescriptions. Le premier article réaffirme la cote de 60 pieds $(19,44 \mathrm{~m})$ pour les grands chemins forestiers, tandis que le deuxième étend cette mesure à l'ensemble des chemins royaux, "bordés hors ledit espace de fossés dont la largeur sera au moins de 6 pieds $[2 \mathrm{~m}$ ] dans le haut, de 3 [1 m] dans le bas et la profondeur de 3 pieds ${ }^{23}$ ". Enfin, les arbres doivent être plantés à 6 et non plus 3 pieds des fossés. La largeur des routes de moyenne importance entre les fossés, identiques aux précédents, est fixée à 36 pieds $(11,66 \mathrm{~m})$. Le profil des chemins est désormais déterminé jusqu'au début du XIX ${ }^{\mathrm{e}}$ siècle et leurs usages se précisent : la chaussée centrale pavée accueille la circulation rapide et les chaussées latérales un trafic plus lent, de voitures et de chevaux également ${ }^{24}$.

Cet arrêt fait l'objet d'une Instruction aux inspecteurs et ingénieurs des Ponts et chaussées $^{25}$. Les deux catégories de routes principales sont explicitement définies par des cotes : 60 pieds portés à 72 avec les fossés et 36 pieds portés à 48. La hiérarchisation des chemins est confiée à l'intendant qui fera faire un état général et des plans ou projets particuliers pour chaque chemin. L'Instruction de 1738 enfin affine cette hiérarchie : les grandes routes et routes royales n'auront pas moins de 60 pieds entre les fossés, les grands chemins, 48 , les chemins royaux, 36 , les chemins de traverse, 30 pieds, exception faite, comme à l'accoutumée, des chemins de montagne ou traversant les marais dont la largeur peut être réduite. Les grandes routes se rapprochent des avenues de Versailles, du boulevard parisien et des grandes avenues conduisant à la capitale, sans jamais toutefois les excéder. A titre de comparaison, l'avenue de Paris à Versailles occupe 25 toises entre les écuries, le boulevard parisien mesure 16 toises, soit 78 pieds, avec une chaussée pavée de 3 à 4 toises, soit 18 à 24 pieds, contre 20 pieds pour la chaussée des routes les plus larges. Mais c'est bien à ces modèles que les instructions de 1720 et 1738 font référence implicitement : les routes sont plantées et les promenades urbaines correspondent elles aussi souvent à la route, lorsqu'elle est forcée de contourner la ville, comme à Limoges.

22. Ibid., p. 240.

23. Ibid., p. 247.

24. Guy Arbellot, Atlas de la Révolution française, op. cit., p. 768. L'auteur fait référence à un mémoire inédit de Perronet conservé à l'École des Ponts et chaussées.

25. Hubert Gautier, Traité de la construction des chemins, op. cit., p. 249-251. 


\section{Plantation et clôture des chemins}

La plantation d'arbres le long des grands chemins est une obligation fort ancienne. L'article 356 de l'Ordonnance de Blois de mai 1579 prescrivait, que " tous les chemins [seraient] réduits à leur ancienne largeur, nonobstant toutes usurpations, par quelques laps de temps qu'elles puissent avoir été faites, et à ce que ciaprès n'y soit fait aucune entreprise, [seraient] plantés et bordés d'arbres, comme ormes et noyers, ou autres selon la nature et commodité du pays, au profit de celui auquel la terre prochaine appartiendra ${ }^{26}$ ”. Les arbres, propriété des riverains, ont donc pour fonction de délimiter la route et les terrains qui la bordent, donnant ainsi à voir le tracé de la route comme la grandeur des héritages environnants, et, par leur solidité et leur grande visibilité, de rendre impossible toute usurpation du domaine public. L'édit de janvier 1583, portant règlement pour les Eaux et Forêts, les chemins et les rivières, confirmait l'obligation de planter, "à la distance de 24 pieds $[7,77 \mathrm{~m}]$ ", d'un arbre à l'autre ${ }^{27}$.

Comme le rappelle l'Instruction aux inspecteurs et ingénieurs des Ponts et chaussées pour l'exécution de l'arrêt du 3 mai 1720, ce texte "a deux objets; le premier, la largeur des chemins, le deuxième, la plantation d'arbres sur les bords des grands chemins ${ }^{28}$ ". L'arrêt prescrit que la nécessité de "repeupler le royaume d'ormes, hêtres, châtaigniers, arbres fruitiers et autres ${ }^{29}$ ", en renouvelant l'obligation faite aux riverains des chemins de planter des arbres en bordure de leurs terres, supposait de déterminer une bonne fois pour toute l'emprise de la route que protège la présence désormais indispensable des fossés. 30 pieds au plus $(9,72 \mathrm{~m})$ doivent séparer chacun des arbres dont le choix est fonction de la nature du terrain. L'application du texte est désormais assurée par la possibilité donnée aux seigneurs voyers de planter à leurs frais et pour leur profit sur tout héritage. En outre, les arbres qui périraient doivent être remplacés. L'instruction contient une mesure complémentaire : la pose de bornes à l'extérieur des fossés fera l'objet d'un procès-verbal remis à l'intendant « de manière que les propriétaires ne puissent avoir aucun prétexte de retarder l'exécution dudit arrêt du 3 mai 1720 , quant aux trous qu'ils doivent faire pour planter des arbres et à la plantation desdits arbres ${ }^{30}$ ".

Si les plantations marquent l'emprise de la route sur le territoire, elles contribuent de surcroît à son agrément, parce qu'elles soulignent la rectitude des voies, mais aussi par la beauté des arbres et l'ombrage qu'ils procurent, pour les piétons en marge de la chaussée qui doit demeurer au soleil pour éviter boue et verglas. Dès le XVII ${ }^{e}$ siècle, les théoriciens du jardin soulignaient l'intérêt de ces alignements. En 1638, Jacques Boyceau de la Barauderie affirmait que l'allée d'ormes du jardin des Tuileries était une des plus belles que l'on puisse observer par sa largeur, 
30 pieds, proportionnée à sa longueur ${ }^{31}$. Il en déduisait que «les longues routes et allées des campagnes, si elles passent trois à quatre cent toises de long [580 à $780 \mathrm{~m}$ environ], en doivent avoir sept à huit toises [ 42 à 48 pieds, soit 15,28 à 17,47 m] de large pour être belles et magnifiques, et doivent être plantées à double rang de chaque côté ». Ces allées de 42 à 48 pieds de large devaient pourtant être bien inférieures par leur majesté aux routes royales forestières portées à 72 pieds dès 1607, largeur réaffirmée par l'Ordonnance des Eaux et forêts de 1669, puis adoptée pour toutes les grandes routes en 1720. Gautier parlait aussi en 1693 de l'ornement des chemins; à propos des chemins privés, il indiquait qu'ils étaient "différemment ornés, comme par de simples haies, quelquefois par une pelouse, d'autres fois par un simple sillon, souvent par des pierres dressées et rangées de champ comme dans un parterre, quelquefois assises en forme de mur et de parapin [sic] ", tandis que les chemins de traverse étaient "bordés d'une haie, d'un mur ou d'une palissade, etc., pour préserver le contenu des champs voisins ${ }^{32}$ ". Le verbe orner semble abusif pour désigner de simples haies mais la beauté des alignements d'arbres est goûtée de tous au XVII ${ }^{e}$ comme au XVIII ${ }^{e}$ siècle. Cassini lui-même, dans l'Avertissement ou introduction à la carte générale et particulière de la France, publiée après 1782 , exprimera le regret de n'avoir pu rendre compte par le dessin de la transformation des campagnes et de l'embellissement du paysage qui se produisaient au moment où ses ingénieurs étaient occupés à lever la carte du royaume, à partir de 1750 : «En effet, quelle variété [changement] ne remarque-t-on point tous les jours dans le plan, la vue et les environs d'un pays ? Ici ce sont des forêts là où l'on ne voyait que des terres labourables; là ce sont des grands chemins ornés de belles avenues, où l'on passait par des précipices impraticables aux voyageurs; ici mille objets nouveaux forment des points de vue agréables, où on ne découvrait que des montagnes et un horizon couvert de tous côtés; là de nouveaux ruisseaux ou canaux viennent arroser un pays, et procurent l'abondance, où l'éloignement des rivières et la sècheresse rendaient un pays misérable et désert? Combien de châteaux anciens ont été détruits reconstruits dans les positions convenables, pour que le bâtiment puisse symétriser avec tout ce qui l'environne ${ }^{33}$. "Nombreux sont ceux qui partagent la vision paysagère du célèbre cartographe. Il est peu commode d'évaluer la couverture réelle du territoire en matière de plantations faites depuis 1720 puis après 1738 ; en revanche, il serait possible d'observer le zèle que les intendants mettent à faire appliquer ses règlements. Pour la généralité de Guyenne, Tourny fut forcé de rédiger une nouvelle instruction le 24 décembre 1743, puis une ordonnance le 25 juillet 1744, pour réaffirmer l'obligation de planter faite aux propriétaires riverains, "soit des arbres fruitiers tels que noyers, châtaigniers, poiriers,

31. Jacques Boyceau de la Barauderie, Traité du jardinage, Paris, 1638, livre IV, ch. 4, p. 72-73.

32. Hubert Gautier, Traité de la construction des chemins, op. cit., p. 14.

33. César-François Cassini de Thury, Avertissement ou introduction à la carte générale et particulière de la France, s.l.n.d. [après 1782], p. 9-10. 
pommiers, pruniers, merisiers, mûriers, etc., soit des arbres à ouvrage, tels que chênes, ormes, hêtres, frênes ou autres ", et préciser la grandeur des trous à creuser et la taille à pratiquer avant croissance ${ }^{34}$.

\section{Carrefours, ponts, portes et rues}

L'ornement des carrefours forestiers est une recommandation fort ancienne également : l'article 6 de l'édit de juillet 1607 en donnait le détail. Ordre était donné par le roi "que dans les angles, ou coin des places croisées, triviaires ou bivioires [sic], qui se rencontreront es routes et chemins royaux des forêts, nos officiers, des maitrises seront planter incessamment des croix, poteaux ou pyramides à nos frais, es bois qui nous appartiennent, et pour les autres, aux frais des voisins et intéressés, avec inscriptions et marques apparentes du lieu où chacun conduit ${ }^{35}$ ". Toutes les intersections ne sont pas concernées, puisqu'il s'agit des routes forestières; l'article 2 portait lui aussi sur les nouveaux chemins à faire "pour la facilité du commerce et la sureté publique ${ }^{36}$ " dans les forêts, et plus particulièrement celles du roi. Sur les cartes des Atlas de Trudaine conservés aux Archives nationales ou sur les cartes routières établies par les ingénieurs des Ponts et chaussées, conservées dans les séries $C$ des archives départementales, on observe effectivement quelques carrefours forestiers dotés de pyramides ou de colonnes, généralement nommés " croix du Grand Maitre ", comme sur une Carte pour servir au projet de la route de Sézanne à Nogent-sur-Seine, levé en 1770 par le sieur Morla, géographe des Ponts et chaussées, figurant le détail des routes traversant la forêt de la Traconne ${ }^{37}$. Il serait opportun et commode de faire l'inventaire de ces constructions signalées également dans les descriptions ou itinéraires du royaume. Plusieurs sont élevées pour marquer la limite de deux généralités, dans des carrefours, comme l'obélisque de Villeneuve-le-Comte aux environs de Coulommiers, ou même sur des ponts, comme celui de Nuits-sous-Ravière entre Champagne et Bourgogne, sur l'une des routes de Paris à Lyon, entre Tonnerre et Montbard, élevé en 1738 (ill. 1).

$\mathrm{Ni}$ textes législatifs, ni les ouvrages techniques ne contiennent de prescription quant à la décoration des ponts. On connaît pourtant quelques pyramides destinées à marquer le milieu du parcours, comme à Blois (1716) et La Charité (1731) sur la rivière de Loire ou encore à Moulins où le pont de Règemortes franchit l'Allier, et on sait le soin apporté par Émiland-Marie Gauthey à la décoration des piles et des tabliers de plus modestes ponts de Bourgogne, comme celui jeté sur la Thalie

34. Michelle Lhéritier, L'Intendant Tourny (1695-1760), Paris, Librairie Félix Alcan, 1920, t. II, p. 165. 35. Hubert Gautier, Traité de la construction des chemins, op. cit., p. 215.

36. Ibid., p. 213.

37. Arch. dép. de la Marne, C 4031, nº 27. 


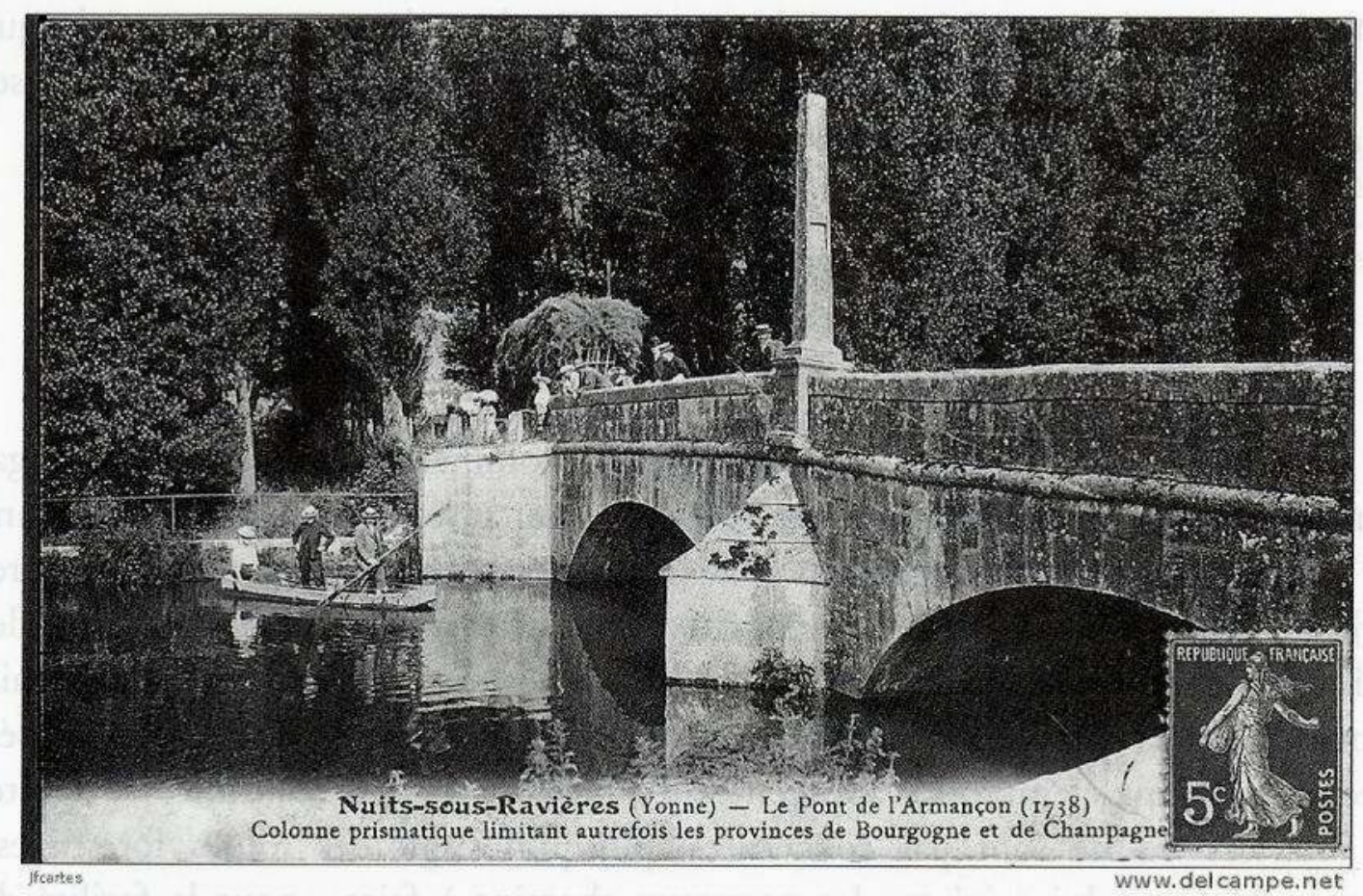

Ill. 1 : Pyramide du pont de l'Armançon entre Nuits et Ravières, dans l'Yonne, établie en 1738 pour délimiter les provinces de Champagne et de Bourgogne sur la route de Paris et Lyon par Tonnerre et Montbard, carte postale du début du XXe siècle, coll. part. Cl. de l'auteur.

à Châtenoy-le-Royal $(1766)^{38}$. Le pont présente d'ailleurs deux faces susceptibles de décoration: on le franchit par la route mais on le passe aussi par voie d'eau où le trafic est bien plus intense. Ainsi, la chaussée au-dessus mais aussi les faces extérieures des parapets et les piles au-dessous peuvent donner lieu à un traitement particulier. L'ouvrage le plus spectaculaire est le pont jeté sur aux Belles-Fontaines de Juvisy, sur la route royale de Paris à Lyon, achevé en 1728 et plusieurs fois décrit au XVIII ${ }^{e}$ siècle $^{39}$. Il forme un écrin à la route en ce sens où il sert de halte durant laquelle on peut observer la longue perspective formée par les arbres et l'aménagement de l'espace de la chaussée (ill. 2). Pour les ponts comme pour les carrefours, les documents sont nombreux et partiellement exploités au regard de l'histoire de la construction; en revanche, les liens qu'ils entretiennent avec leur environnement restent à examiner plus largement que la simple étude de l'ensemble urbain pont-traverse urbaine-quais dont l'unité semble évidente, comme à Orléans

38. Fernand de Dartein, Études sur les ponts en pierre remarquables par leur décoration, antérieurs au XVIII e siècle, Paris, Ch. Béranger, 1907-1909, 4 vol. ; Anne Coste, Antoine Picon et Francis Sidot dir., Un Ingénieur des Lumières: Émiland-Marie Gauthey, Presses de l'École nationale des Ponts et chaussées, 1994, $379 \mathrm{p}$.

39. Louis Brunel, "Juvisy au XVIII" siècle. Le détournement du pavé royal de Lyon... ", Mémoires et documents de la Société historique et archéologique de Corbeil d'Étampes et du Hurepoix, numéro spécial, t. XI, 1975, p. 31-32; Mercure de France, août 1730, p. 1784-1785; Antoine-Nicolas Dézallier d'Argenville, Voyage pittoresque des environs de Paris, Paris, 1755, p. 202 ; Pierre-ThomasNicolas Hurtaut, Dictionnaire historique de la ville de Paris et de ses environs, Paris, 1779, t. III, p. 345 ; Jacques-Antoine Dulaure, Nouvelle description des environs de Paris, Paris, 1786, t. I, p. 259. 


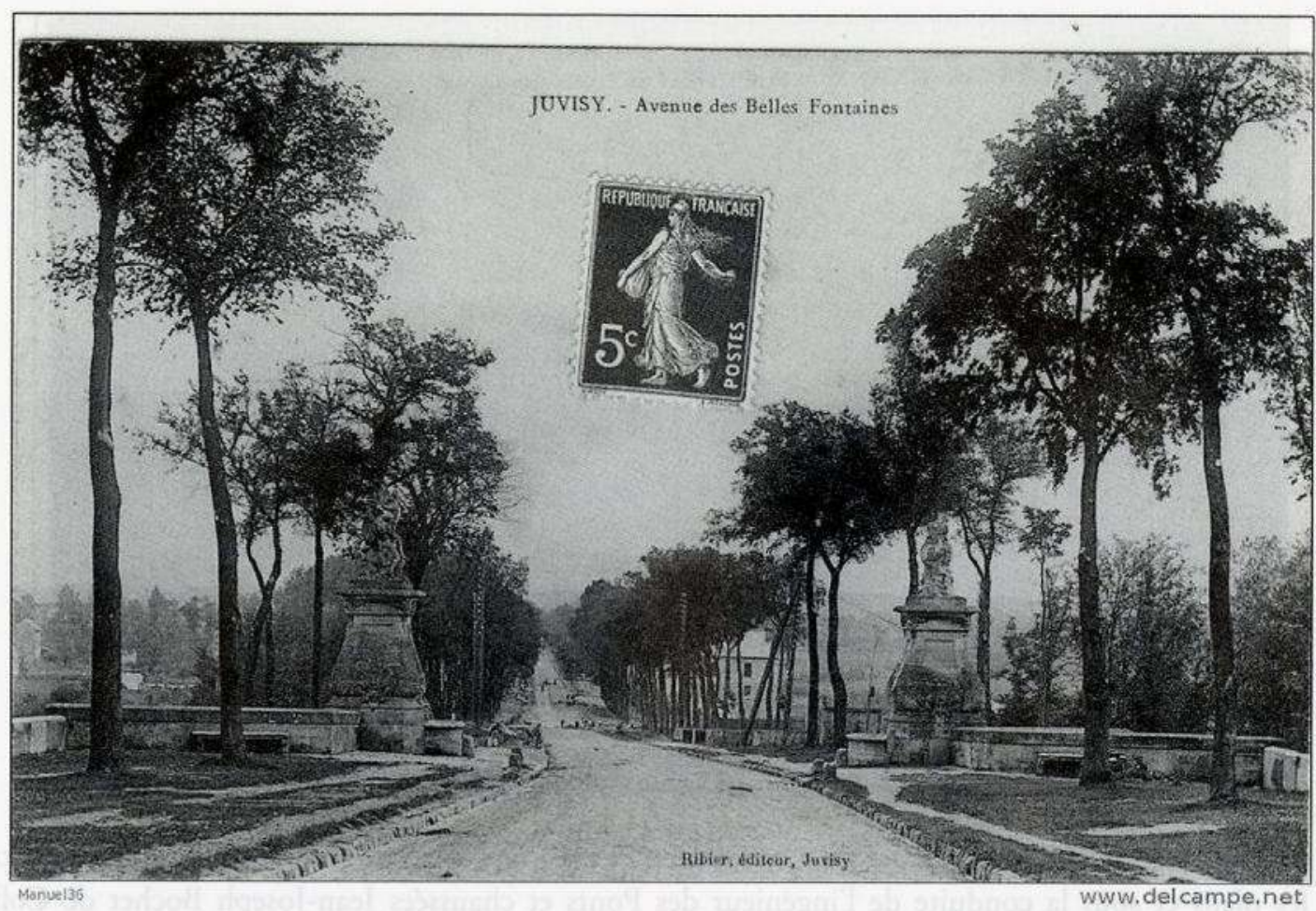

Ill. 2 : Pont des Belles-Fontaines à Juvisy, ouvrage commencé en 1728, décoré de deux fontaines monumentales dont l'une porte un médaillon figurant le roi ; les arbres ont été replantés sur les bermes, selon la loi du 9 ventôse an XIII, et non au-delà des fossés comme au XVIII ${ }^{e}$ siècle, carte postale du début du XXe siècle, coll. part. Cl. de l'auteur.

et à Tours ${ }^{40}$. Le projet du "nouveau faubourg dénommé Bernage » et de la traverse et nouvelle place centrale de la ville représenté par Règemortes sur la deuxième planche de sa Description du nouveau pont de pierre construit sur la rivière d'Allier à Moulins, publiée en 1771, est loin d'être unique ${ }^{41}$. La construction d'un pont est si coûteuse qu'elle engendre souvent des projets urbains à la mesure de l'effort consenti par le roi.

Comment dès lors imaginer que ces nouveaux chemins et ces ponts viennent butter contre de solides et monumentales portes médiévales dont la fonction principale est l'obstruction? Si la porte demeure une limite, matérialisée par un rétrécissement du passage entre deux piles, elle sera désormais précéder d'une place et sa reconstruction s'accorde avec le redressement voire l'élargissement de la rue. L'architecture des portes et leurs dimensions vont s'en trouver modifiées. Si la rue n'est pas alignée dans le prolongement de la route, la porte comble simplement l'espace existant entre les maisons; si, au contraire, l'alignement vise à élargir le commencement de la rue pour former une sorte de place, la porte s'en trouve

40. A propos du pont et de la traverse de Tours notamment, voir François Dumas, La Généralité de Tours au XVIII siècle. Administration de l'intendant Du Cluzel (1766-1783), Mémoires de la Société archéologique de Touraine, t. XXXIX, Tours, L. Péricat, 1894, p. 219-236; la largeur de la rue est portée à 42 pieds 6 pouces, largeur du pont entre les deux parapets.

41. Louis de Règemortes, Description du nouveau pont de pierre construit sur la rivière d'Allier à Moulins, Paris, Lottin l'Aîné, 1771, 47 p. et 16 pl. ; voir pl. II et explication p. 8. 


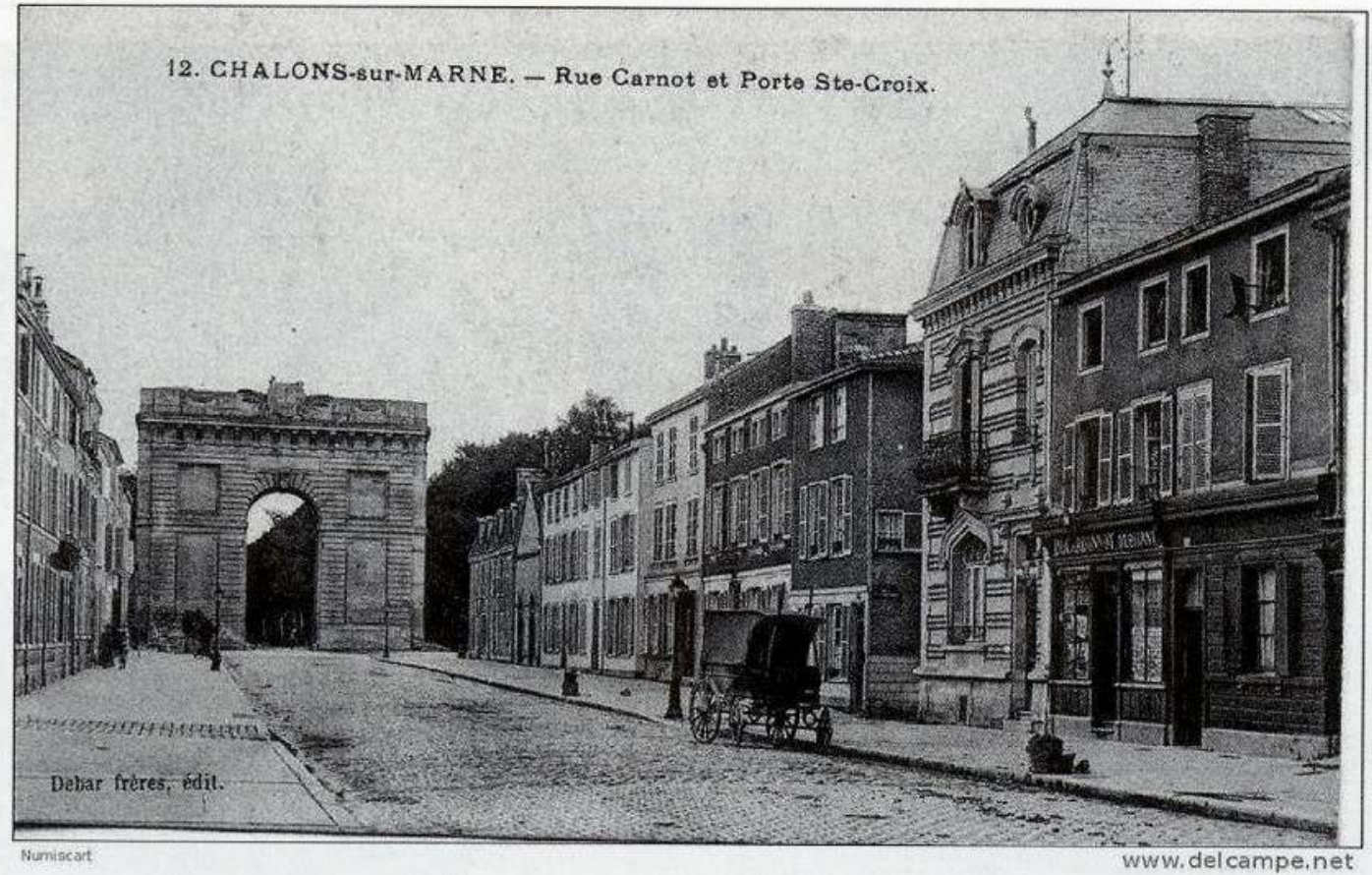

Ill. 3 : Porte Sainte-Croix à Châlons-en-Champagne et rue conduisant à l'intendance, édifice construit sur les plans et sous la conduite de l'ingénieur des Ponts et chaussées Jean-Joseph Bochet de Colluel entre 1769 et 1771, carte postale du début du XXe siècle, coll. part. Cl. de l'auteur.

agrandie; l'arche centrale, qui peut prendre des dimensions colossales, comme à Bordeaux, avec les portes de Bourgogne (1751) et d'Aquitaine (1746), est flanquée de murs ou de grilles, de portes piétonnes. La porte de Bourgogne ne mesure pas moins de 17 toises et 2 pieds soit 115 pieds, largeur qui correspond au cours qui aboutit au port et à la nouvelle place semi-circulaire dont elle constitue l'ornement principal; l'arc triomphal occupe 10 toises, ou 60 pieds $^{42}$, la mesure des plus grandes voies romaines ${ }^{43}$. Si sa construction est le fait d'un évènement particulier et exceptionnel, comme le passage de la future dauphine, Marie-Antoinette d'Autriche, le 11 mai 1770 à Châlons-en-Champagne, la porte est aussi triomphale (ill. 3 et 4). Comme la grande route de Paris en Allemagne qui parvient à cette porte depuis l'Est et constitue l'entrée principale de la ville, la porte Sainte-Croix adopte des dimensions bien supérieures à l'ordinaire, qui seront bientôt celles de la rue qui conduit à la nouvelle intendance achevée en $1764^{44}: 60$ pieds soit 10 toises de largeur, plaçant cet arc entre les portes parisiennes Saint-Denis et Saint-Martin larges de 73 pieds 9 pouces pour la première et 53 pieds 7 pouces pour la seconde ${ }^{45}$. Jacques-François Blondel, chargé de célébrer le retour du Bien-

42. Arch. dép. Gironde, C 1164.

43. Voir aussi Charles Duclos, Essais sur les Ponts et chaussées. La voirie et les corvées, Amsterdam, Chatelain, 1759 ; seconde partie, chapitre premier, "Des différentes largeurs des chemins », p. 134171.

44. Arch. dép. de la Marne, C 4023, n 3, «Plan d'une partie de la rue Sainte-Croix, de la nouvelle porte et des bâtiments qui l'accompagnent ", 23 décembre 1769, Coluel, ingénieur en chef de la province et frontière de Champagne.

45. Jacques-François Blondel, L'Architecture française, Paris, Charles-Antoine Jombert, 1756, t. III, p. 10 et 14. 


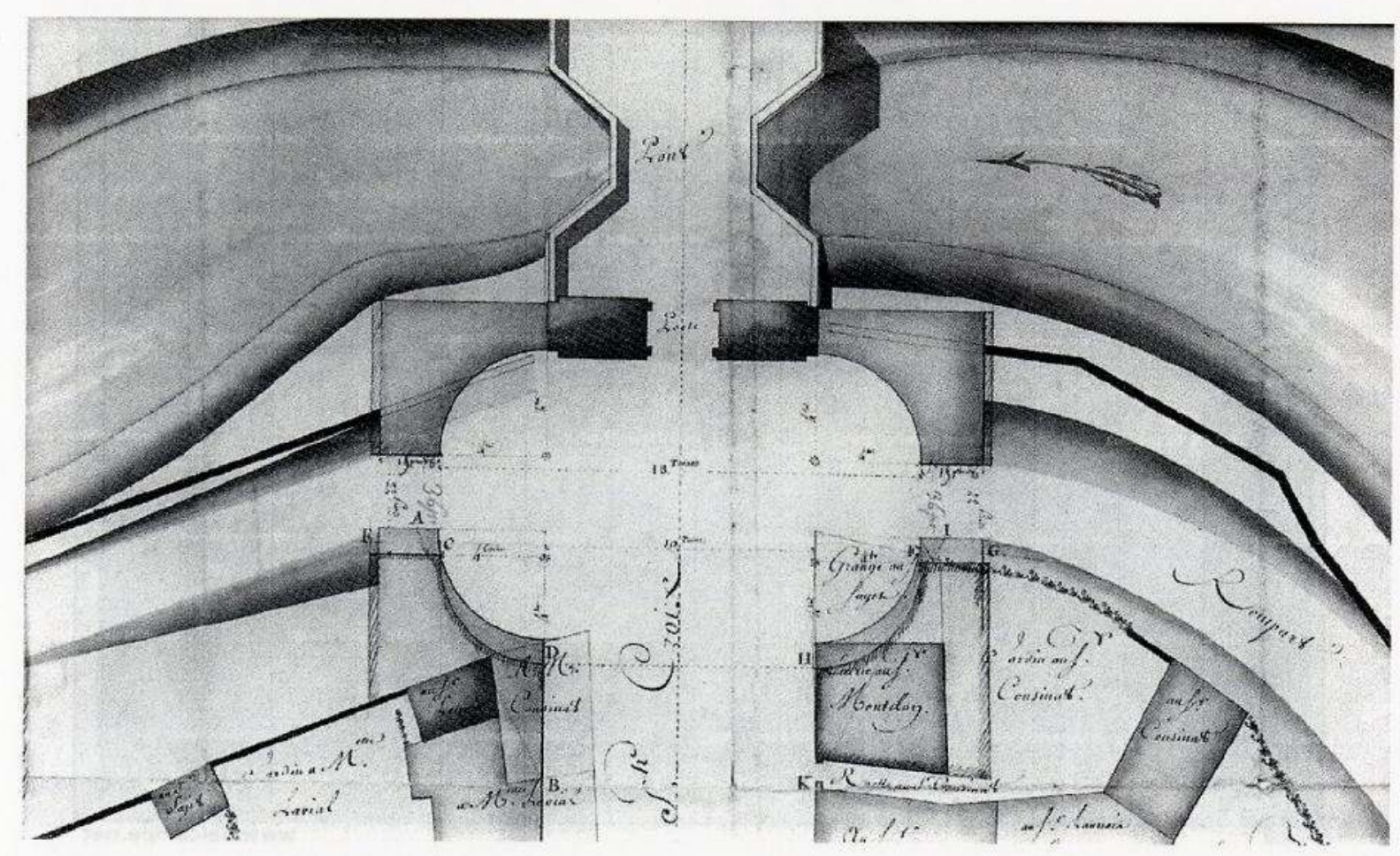

Ill. 4 : Bochet de Coluel, plan masse de la porte Sainte-Croix et de la place projetée au départ de la rue du même nom, entrée sud-est de la ville par la route de Vitry-le-François, 23 décembre 1769. Arch. dép. de la Marne, C 4023, 3, détail. Cl. de l'auteur.

Aimé à Paris après la maladie de Metz, avait déjà adopté, sept ans après l'Instruction de 1738 , la cote fixée pour les grandes routes royales; il faisait mention de la commande d'un arc éphémère dans une note accompagnant la description de la porte Saint-Denis : "(b). Le 7 septembre 1745, au retour de Louis XV de l'armée de Flandres, la Ville de Paris fit ériger un arc de triomphe, peint sur toile et monté sur un bâti de charpente des deux côtés de cette porte : cette décoration avait de largeur 72 pieds sur 87 de haut, y compris un amortissement posé sur un attique et soutenu du côté du faubourg sur un ordre de colonnes ioniques de marbre coloré et les ornements rehaussés d'or. Le côté de la Ville était décoré d'une manière plus rustique, et surmonté de même par un grand amortissement, supportant des allégories et des attributs relatifs au sujet; ces décorations, dont j'ai données les dessins, furent exécutées avec succès par les sieurs Tremblin et Labbé, peintres de la Ville ${ }^{46}$. " L'arc triomphal de Blondel occupait en 1745 la largeur idéale et non réelle de la grande route supposée élargie, dans sa dernière portion, conduisant à la capitale depuis Saint-Denis. En effet, entre la grande avenue plantée d'une double rangée d'arbres qui s'étendait devant la basilique et la porte SaintDenis, la rue du faubourg éponyme ne présentait à cette date aucune régularité, ni par son tracé, ni par sa largeur. Pourtant la cote de 72 pieds ou 12 toises s'était imposée à Blondel et devait être adoptée pour les nouveaux monuments.

Dans les années 1740-1750, de nombreux projets, quelques édifices triomphaux et surtout de nombreuses portes tripartites formées de piles et de grilles sont

46. Ibid., p. 14 . 


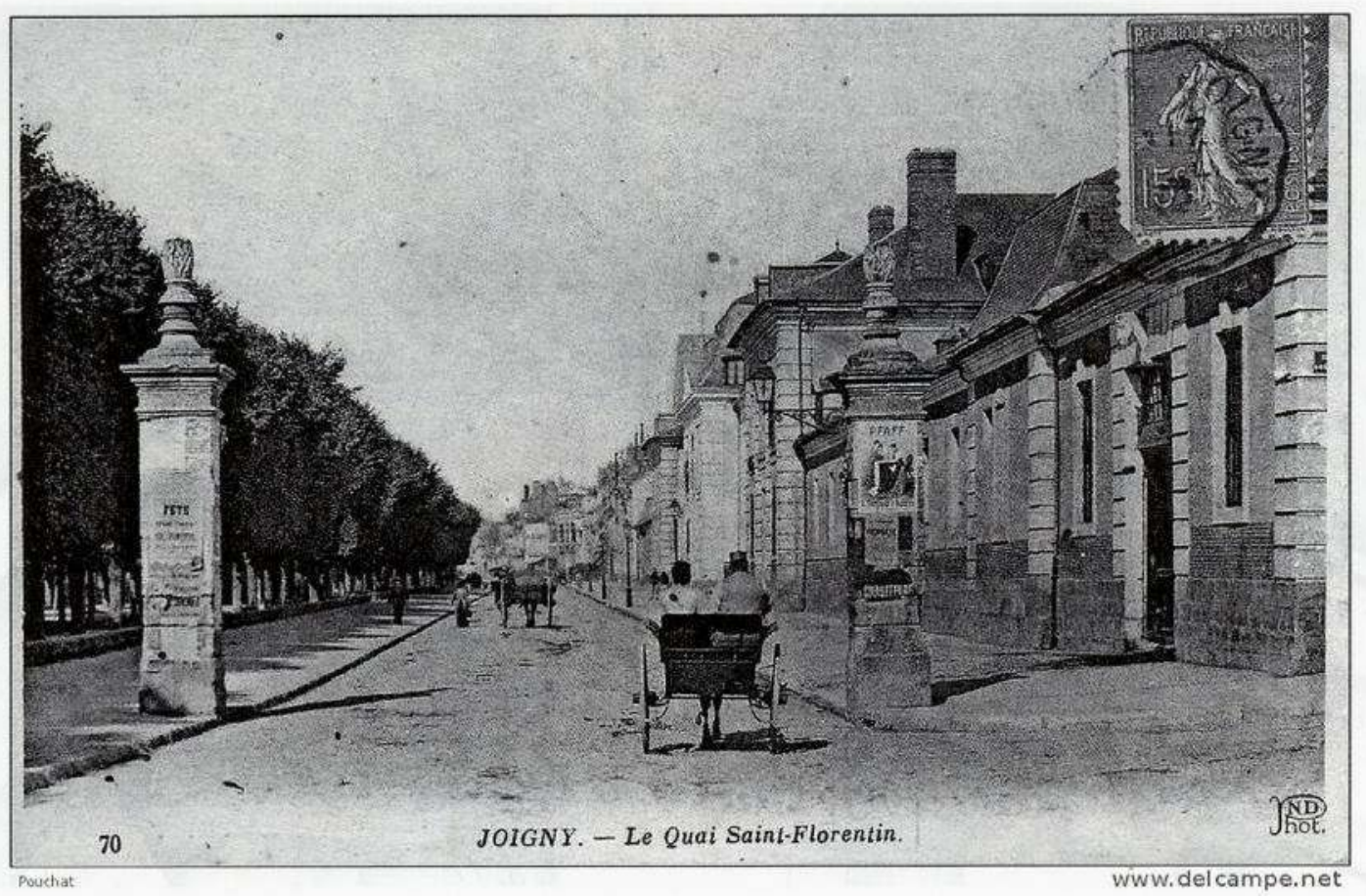

Ill. 5 : Cours Saint-Florentin à Joigny, dans l'Yonne, porte sud de la ville, sur la route de Paris à Lyon par Sens et Auxerre, carte postale du début du XXe siècle, coll. part. Cl. de l'auteur.

dessinées pour servir de perspective à de nouvelles chaussées qui commandent aussi l'élargissement et le redressement d'une rue principale. À Joigny, sur l'une des routes bourguignonnes de Paris à Lyon, entre Sens et Auxerre, des piliers supportant des grilles matérialisent les limites de la ville à l'extrémité du quai de Paris comme du cours Saint-Florentin de l'autre côté, en bordure de l'Yonne (ill. 5). Le phénomène d'ouverture est précoce, tout comme la levée des plans d'alignement, et les conséquences sur la manière d'embellir importantes. Jean-François Legendre figure au premier rang parmi les acteurs de ce renouveau. S'il ne publie qu'en 1765 sa Description de la place Louis XV que l'on construit à Reims, des ouvrages à continuer aux environs de cette place et de ceux à faire dans la suite pour l'utilité et l'embellissement de cette ville et, en 1769 le Plan général de la ville de Reims correspondant ${ }^{47}$, voilà dix ans qu'il travaille à cette entreprise de modernisation de la ville du sacre, depuis la levée du plan général d'alignement de la ville commencée en 1755. De très nombreux plans conservés dans la série $\mathrm{C}$ des archives départementales de la Marne, pour la généralité de Champagne où il est ingénieur entre 1743 et 1763, permettent de se rendre compte de la globalité du projet qui comprend l'alignement général, la construction de la place royale et ses abords, celle de portes monumentales et l'aménagement du tour de ville, de promenades, de nouveaux carrefours et des routes qui y conduisent. Sur l'un d'eux, daté de 1750, figurent, surajoutés

47. Par le sieur Legendre, ingénieur du roi, inspecteur général des Ponts et chaussées de France, chargé par le conseil de la direction desdits ouvrages, Paris, Impr. De Prault, 1765, 17 p. et 8 pl.; rééd., Paris, Lattré, 1769. 
en rouge, les plans des portes projetées dont il donnera l'élévation dans les vignettes du plan de 1769 (ill. 6 et 7): leurs dimensions s'accordent avec celle des avenues bientôt plantées dont elles fermeront la perspective.

\section{Des bonnes routes aux belles routes}

Il faut donc s'imaginer à terme, après quelques dizaines d'années de labeur des hommes et de croissance des végétaux, de grandes routes larges comme les avenues des châteaux, qui la prolongent jusqu'aux plus antiques ou belles demeures, de longues perspectives bordées d'arbres se succédant d'une ville à l'autre, des carrefours en patte d'oie ou en ronds-points, plantés pour souligner leur forme, pour les plus importants occupés par une croix ou une pyramide en leur centre, indiquant les directions ou commémorant un évènement, des chaussées pavées franchissant les ponts dont le parapet est parfois orné d'un monument identique à ceux des carrefours, dont le tablier peut s'élargir pour former un belvédère, une halte agrémentée de bancs, comme à Juvisy, sur la route royale de Paris à Lyon. S'il paraît évident qu'un pont engendre la création d'une place, voire le percement d'une rue, comme à Orléans, Tours ou Mantes, il l'est moins qu'une route commande une porte, comme à Bordeaux, Châlons-en-Champagne ou encore Dijon (porte Condé ou Guillaume, 1786). Ainsi, en confiant aux ingénieurs des Ponts et chaussées la modernisation des routes, l'occasion leur est également donnée de produire systématiquement un avis, voire d'initier la transformation des abords de la ville comme de la ou des rues principales, selon un principe de continuité territoriale. Prenant leurs ordres du contrôle général, tout en travaillant sur le terrain au côté des intendants, ils se trouvent dans la situation d'agir rapidement et de manière cohérente, dehors comme dedans. En 1920, Michel Lhéritier, auteur d'un ouvrage consacré à Tourny, intendant du Limousin puis de Guyenne, écrivait : "Tout se tient dans l'aménagement du pays, tel que Tourny l'entend : les villes y ont leur part aussi bien que les rivières et les routes; la même ordonnance est de mise pour celles-là, comme celles-ci; il convient que les uns cadrent avec les autres. Sans qu'il s'attarde jamais à dégager le sens des retouches qu'il apporte, l'intendant traite l'ensemble géographique, comme s'il était averti de son essentielle unité. Le dessin des villes se modifie pour répondre au nouveau dessin des routes, tracées elle-même sur le canevas des rivières ${ }^{48}$."

Une chronologie affinée des transformations urbaines, repérées pour les villes principales par Louis Hautecœur dans son Histoire de l'architecture classique en France, doit être enrichie de réalisations plus modestes mais non moins modernes mais aussi de l'examen des textes réglementaires, des correspondances entre Paris et la province, entre les contrôleurs généraux des finances successifs et les intendants - on ne retient trop souvent que le terme des projets sans considérer la genèse que les lois et la correspondance renseignent. Si les premiers textes des architectes

48. Michel Lhéritier, L'Intendant Tourny (1695-1760), Paris, Librairie Félix Alcan, 1920, t. II, p. 188. 
et des amateurs consacrés à la ville ne sont publiés que les années 1750 et 1760 - Laugier en 1753, Poncet de la Grave en 1755, Patte en 1765 et 1769 -, les prescriptions qu'ils contiennent ont déjà été formulées voire mises en œuvre par les grands serviteurs de l'État et quelques édiles en province, avant 1750. De même, si l'architecture publique connaît un renouveau important dans la seconde moitié du siècle, il faut chercher avant l'arrivée de Marigny à la direction des Bâtiments du roi en 1751 le ferment des idées et les moyens qui vont faire de la France un territoire moderne et embelli. Le 24 mars 1738, année de l'Instruction, année où commencent les travaux de la percée de Limoges depuis la route de Lyon jusqu'au cœur de la ville, les élèves de l'Académie royale d'architecture, travaillaient pour l'obtention du Grand Prix au dessin d'une " entrée ou plutôt d'une porte pour une grande ville, entourée d'un fossé de 20 toises de largeur, avec un pont pour traverser le fossé et dans la ville une rue qui réponde à la magnificence de l'entrée ". L'examen des dessins, le $1^{\text {er }}$ septembre, révèle qu'il y a eu méprise sur la nature du programme : "comme la plus grande partie des élèves qui ont travaillé ne se sont pas renfermés dans le sujet proposé par l'Académie et qu'ils ont fait des arcs de triomphe au lieu de porte de ville qu'on leur avait donné à faire pour une grande ville, la compagnie a été d'avis [...] de faire recomposer tous les élèves pour une porte de ville entourée d'un fossé de 20 toises de largeur avec un pont pour le traverser, déclarant la compagnie qu'elle ne recevra pas les dessins d'arcs de triomphe qui seront proposés. L'édifice de la porte aura entre 18 et 22 toises de face, tout compris, sur une échelle de 2 pouces pour toise ${ }^{49}$. " L'association du pont à la porte était bien celle de la chaussée au monument, soit celle de la route à la ville, conjointement embellies.

Dominique MASSOUNIE maître de conférences université Paris-Ouest-Nanterre-La Défense

49. Henry Lemonnier, Procès-verbaux de l'Académie royale d'architecture, t. V: 1727-1743, Paris, Édouard Champion, 1918, p. 229 et p. 238-239. 